\title{
Clinical application of computed tomographic angiography in patients with renal arterial hemorrhage: Diagnostic accuracy and subsequent therapeutic outcome
}

\author{
QIQI MAO ${ }^{1}$, BAISHU ZHONG ${ }^{2}$, YIWEI LIN ${ }^{1}$, CHAOJUN WANG $^{1}$, WENJIE LIANG ${ }^{2}$, \\ FUQING TAN $^{1}$, BOHUA SHEN $^{1}$ and LIPING XIE ${ }^{1}$ \\ Departments of ${ }^{1}$ Urology and ${ }^{2}$ Radiology, The First Affiliated Hospital, School of Medicine, \\ Zhejiang University, Hangzhou, Zhejiang 310003, P.R. China
}

Received August 23, 2014; Accepted May 15, 2015

DOI: $10.3892 /$ etm.2015.2535

\begin{abstract}
The aim of the present study was to determine the accuracy of multi-section computed tomographic angiography (CTA) for the diagnosis of renal arterial $\mathrm{h}=$ emorrhage in comparison with digital subtraction angiography (DSA) and the effect of diagnostic outcome on therapeutic patient management. A retrospective analysis was conducted of the records of all patients undergoing DSA for acute renal hemorrhage between August 2005 and June 2014. A total of 74 patients were identified in whom CTA was performed prior to the DSA. DSA detected a renal arterial lesion in 68 cases and no bleeding was demonstrated in 6 cases. CTA correctly identified the bleeding site in 59 of the 68 DSA-positive cases. There were 14 patients with negative CTA results that underwent DSA after 2-10 days because of persistent hematuria, and 9 of them were found to have positive DSA results. The sensitivity, specificity, positive predictive value and negative predictive value of CTA for the detection of arterial lesions in the context of acute renal hemorrhage were $0.868,0.833,0.983$ and 0.357 , respectively. All patients with bleeding sites identified by DSA underwent superselective embolization in a single session. Five patients underwent a secondly embolization for recurrent hemorrhage. These results indicate that CTA is an accurate technique for the detection of major arterial hemorrhage and supports its use in the initial examination of these patients.
\end{abstract}

Correspondence to: Dr Chaojun Wang, Department of Urology, The First Affiliated Hospital, School of Medicine, Zhejiang University, 79 Qingchun Road, Hangzhou, Zhejiang 310003, P.R. China

E-mail: urowcj@126.com

Key words: computed tomographic angiography, digital subtraction angiography, pseudoaneurysm, renal hemorrhage

\section{Introduction}

Renal hemorrhage is a life-threatening situation that can be caused by trauma, surgery and biopsy, as well as the sudden spontaneous rupture of renal tumors or aneurysms. Most vein and small artery injuries can be managed by conservative treatment with bed rest, infusion and transfusion therapy (1). However, larger arterial lesions, such as arteriovenous fistula and pseudoaneurysm, often require active surgery or angiographic intervention (2).

Intra-arterial digital subtraction angiography (DSA) remains the gold standard for the diagnosis of renal artery lesions and subsequent superselective embolization has recently emerged as an effective modality for treating arterial injury (3). This procedure, however, is an invasive procedure that carries a risk of serious complications and is burdensome for patients $(4,5)$. For this reason, computed tomographic angiography (CTA), which is minimally invasive, is widely used for diagnostic work-up in patients with suspected renal artery injuries (6). It helps to define the optimal therapeutic strategy (conservative management, open surgery or an interventional angiographic approach) on the basis of the extent and origin of bleeding (arterial or venous). However, this test may delay treatment in cases of massive or persistent hemorrhage, and its diagnostic accuracy for renal artery hemorrhage has not been rigorously studied. The purpose of the present study was to determine the sensitivity and specificity of CTA in comparison with DSA and the effect of diagnostic outcome on therapeutic patient management.

\section{Subjects and methods}

Subjects. The medical records of all patients who had undergone DSA for acute renal hemorrhage at the First Affiliated Hospital of the School of Medicine, Zhejiang University (Hangzhou, China) between August 2005 and June 2014 were analyzed. The present study was approved by the ethics committee of the First Affiliated Hospital of the School of Medicine, Zhejiang University. The patients who underwent multi-section CTA prior to the renal DSA examination were identified. All patients presented with hematuria and signs 
of acute hemorrhage. The clinical data of these patients were evaluated retrospectively.

CTA. CTA examinations were performed using a Brilliance iCT scanner (Philips Healthcare, Cleveland, OH, USA). Contrast material (iopamidol, $370 \mathrm{mg}$ iodine $/ \mathrm{ml}$; Bracco Sine Pharmaceutical Corp. Ltd., Shanghai, China) was administered using a power injector via a $20 \mathrm{G}$ cannula inserted into an antecubital vein. A total of $90 \mathrm{ml}$ contrast material with a flow rate of $5 \mathrm{ml} / \mathrm{sec}$ was injected into each patient. Bolus-tracking was used to trigger data acquisition by placing a region of interest over the abdominal aorta at the level of the 12th thoracic vertebra and setting a trigger threshold of $150 \mathrm{HU}$ above baseline. Scanning parameters were as follows: detector collimation, $128 \times 0.625 \mathrm{~mm}$; pitch, 0.915; tube current, Auto effective mAs; and tube voltage, $120 \mathrm{kV}$. The source axial CT images were post-processed with Advanced Vessel Analysis software on an Extended Brilliance Workspace 4.5 (Philips Healthcare). Three-dimensional reconstructions were performed with multiplanar reconstruction (MPR), volume rendering (VR), maximum intensity projection (MIP) and curve planar reconstruction (CPR). All studies were reviewed by one radiologist in conjunction with another urologist.

$D S A$. The DSA was performed using an Allura Xper FD20 system (Philips Healthcare). A 5F angiographic catheter was introduced into the renal artery via the transfemoral route. Selective renal arteriography was performed when the global angiogram was not able to identify any arterial lesions, or to confirm the bleeding branch before embolic materials were deployed. All procedures were performed by two experienced senior interventional radiologists who were not blinded to the CT angiographic findings for the same patient.

\section{Results}

Patients characteristics. Between August 2005 and June 2014, a total of 88 patients underwent renal angiography for renal hemorrhage. Among them, CTA was performed in 74 patients prior to renal angiography. Table I summarizes the patient characteristics and procedure results. Of the 74 DSA procedures performed, pseudoaneurysm was identified in 45 patients, arteriovenous fistula was identified in 3 patients, and both were found in 10 patients. In addition, 5 patients had renal ateriovenous malformations, with a coexisting arteriovenous fistula in one of them, 1 patient had multiple renal aneurysms, 3 had contrast extravasation, and no active bleeding was identified in 6 patients by renal angiography.

Imaging. The results of multi-section CTA in the detection of renal hemorrhage are summarized in Table II. The sensitivity, specificity, positive predictive value and negative predictive value of CTA for the detection of arterial lesions of renal hemorrhage were $0.868,0.833,0.983$ and 0.357 , respectively. CT angiography correctly identified the site of hemorrhage in 59 of the 68 DSA-positive cases (Fig. 1). These patients immediately proceeded to superselective coil embolization. All patients underwent technically successful embolization. Among them, 57 patients demonstrated complete resolution
Table I. Patient characteristics and procedure results.

\begin{tabular}{lc}
\hline Patient (n) & 74 \\
Age (years) & \\
Mean & 52.2 \\
Range & $26-7$ \\
Gender (n) & \\
Male & 59 \\
Female & 15 \\
Side (n) & \\
Left & 35 \\
Right & 39 \\
Indications for DSA (n) & \\
Post-PCNL & 49 \\
Post-partial nephrectomy & 8 \\
Renal biopsy & 2 \\
Trauma & 2 \\
Post-nephrostomy & 1 \\
Aneurysm & \\
Vascular malformations & 4 \\
Rupture of angiomyolipoma & 10 \\
Idiopathic hematuria & 3 \\
Angiographic findings (n) & 4 \\
Psedoaneurysm & 3 \\
Arteriovenous fistula & 3 \\
Psedoaneurysm and arteriovenous fistula & 3 \\
Aneurysm & 1 \\
Vascular malformation & \\
Vascular malformation and arteriovenous fistula & \\
Free extravasation & \\
No lesion & \\
\hline
\end{tabular}

DSA, digital subtraction angiography; PCNL, percutaneous nephrolithotomy.

Table II. Bleeding site detection by multi-section CTA.

\begin{tabular}{lcc}
\hline & \multicolumn{2}{c}{ DSA } \\
\cline { 2 - 3 } CTA & $\begin{array}{c}\text { Bleeding site } \\
\text { identified }\end{array}$ & $\begin{array}{c}\text { No bleeding } \\
\text { site }\end{array}$ \\
\hline $\begin{array}{l}\text { Bleeding site identified } \\
\text { No bleeding site }\end{array}$ & 59 & 1 \\
\hline $\begin{array}{l}\text { CTA, computed tomographic angiography; DSA, digital subtraction } \\
\text { angiography. Values for CT angiography were as follows: sensitivity }\end{array}$ \\
$\begin{array}{l}\text { 86.8\%, specificity 83.3\%, positive predictive value 98.3\%, negative } \\
\text { predictive value 35.7\%. }\end{array}$
\end{tabular}

of bleeding; however, the bleeding ceased following embolization but subsequently recurred in 5 patients, and these patients underwent a second superselective embolization. 

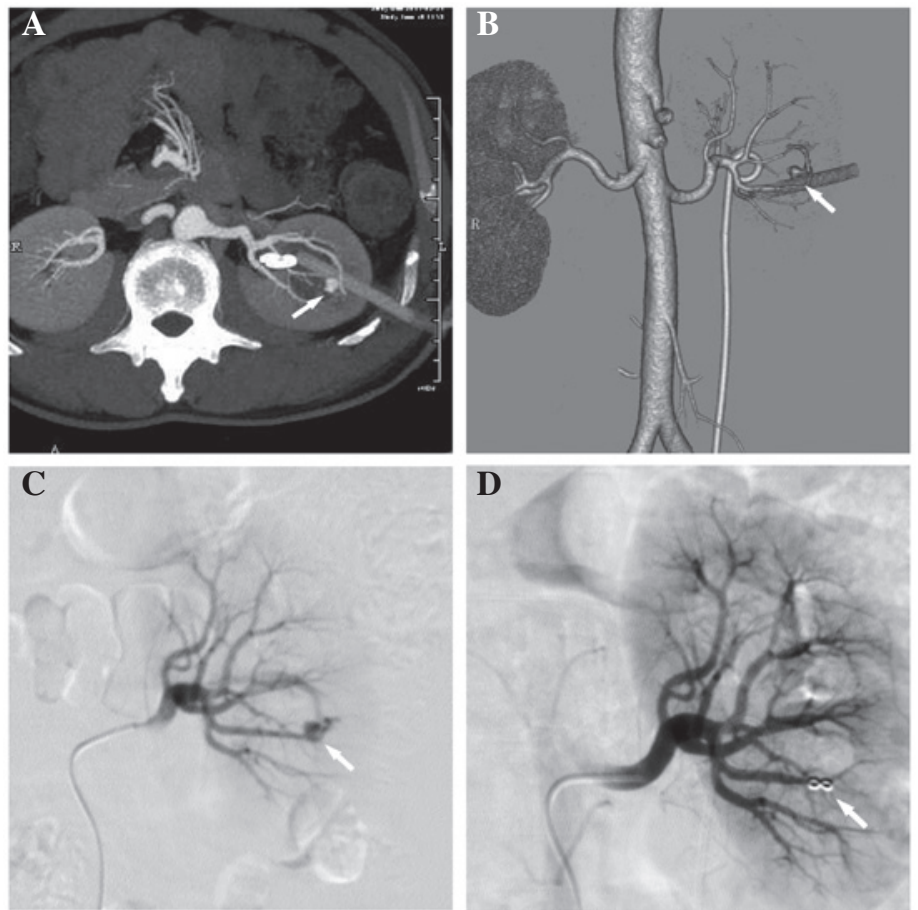

Figure 1. (A and B) Computed tomographic angiography detected a pseudoaneurysm (arrow) in the left lower pole of the kidney following percutaneous nephrolithotomy. Digital subtraction angiography (C) confirmed the lesion and (D) demonstrated occlusion following coil embolization (arrows).
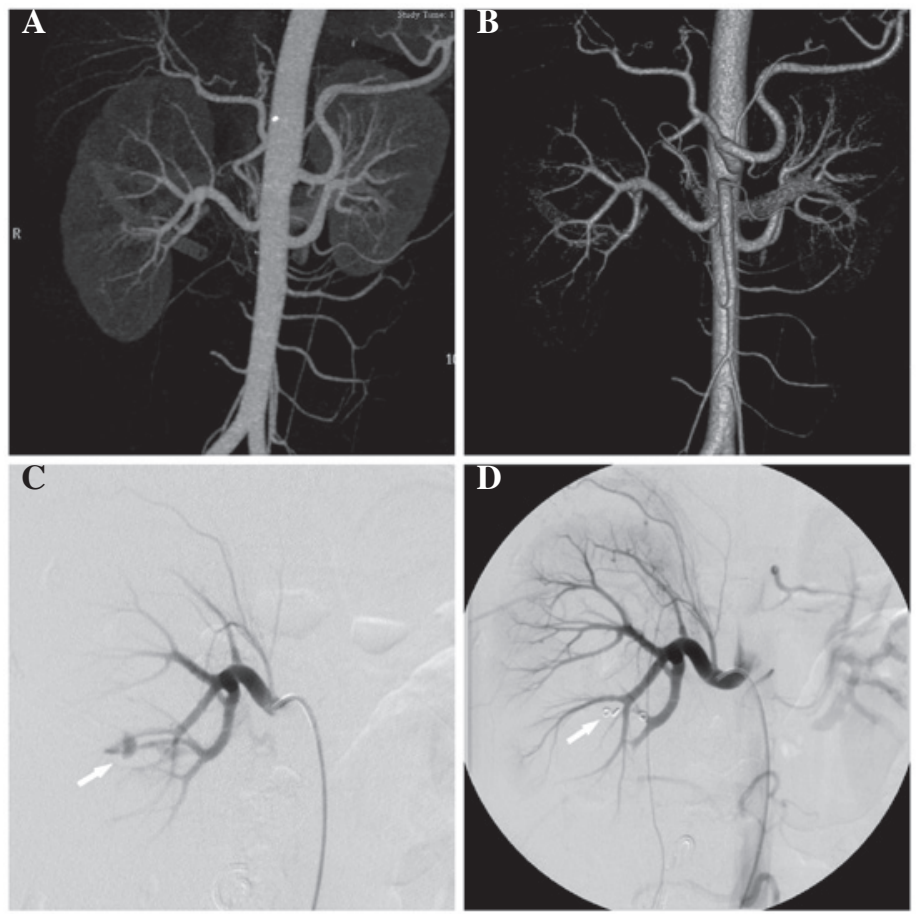

Figure 2. (A and B) Computed tomographic angiography showed a normal right renal artery. Digital subtraction angiography showed (C) a pseudoaneurysm and (D) successful coil embolization (arrows).

Fourteen patients who had negative results on CTA underwent DSA for persistent hemorrhage. Five of them, who were further confirmed to have no lesions by DSA, were finally managed by conservative therapy, while the remaining 9 patients were found to be positive by CTA, and were successfully treated with coil embolization (Fig. 2). The time interval between CTA and catheter angiography for these
9 patients was 2-10 days. In 1 patient, a pseudoaneurysm that was suspected on CTA was not detected on DSA and was considered to be a false positive result (Fig. 3).

Notably, in the remaining 14 patients who underwent DSA directly without preceding CTA because of massive hemorrhage or other reasons, all had arterial lesions and proceeded to successful coil embolization. 

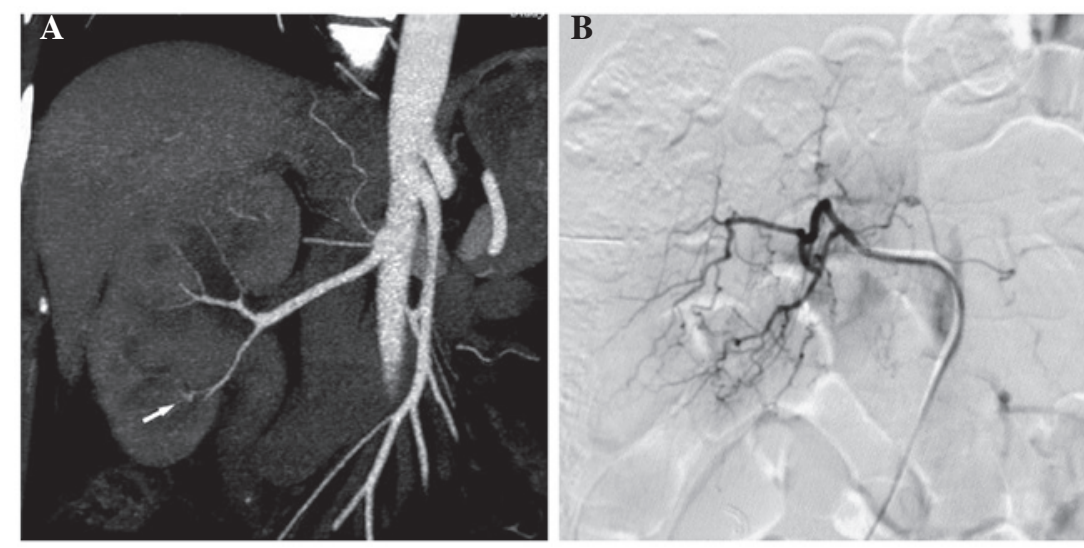

Figure 3. (A) Computed tomographic angiography showed a suspected pseudoaneurysm (arrow) in the right lower pole of the kidney. (B) No arterial lesion was found when examined by digital subtraction angiography.

\section{Discussion}

Renal vascular lesions can be caused by multiple entities such as surgery, biopsy, nephrostomy, trauma, renal mass and vascular anomaly (2). Venous bleeding is self-limiting and can be controlled by conservative measures. Arterial bleeding causing hemodynamic instability is less frequent but necessitates aggressive therapy. Therefore, proper imaging to elucidate the nature of the renal hemorrhage is critical. Although DSA remains the gold standard technique for evaluating renal arterial lesions, the invasiveness of arterial puncture and intra-arterial catheter manipulation is associated with complications such as arterial dissection, distal embolization and groin hematoma, and complications have been reported to occur in $1.3 \%$ of cases (5). CTA provides a rapid, non-invasive imaging modality for diagnosing arterial hemorrhage. The reconstructed radiological images in two or three dimensions can illustrate the morphological findings in a more understandable way, particularly the vessel involved. Over the past few years, CTA has become the most widely used method for the detection and localization of clinically active hemorrhages of an unknown source, and the sensitivity and specificity of CTA is approaching that of digital subtraction angiography in many situations, such as the detection of intracranial cerebral aneurysms, vertebral artery dissection and lower limb angiography (7-9).

However, there are only few reports on the ability of CTA to detect the source of renal hemorrhage in the literature. Sadick et al (10) found that CTA findings were $100 \%$ concordant with transcatheter therapeutic angiography in 7 patients with acute renal hemorrhage. Sommer et al (11) reported that arterial renal hemorrhage was identified by CTA in 42 of 43 patients (98\%). However, these previous studies focused on sensitivity, and did not determine the specificity of CTA in the detection of hemorrhage. In the present study, nine arterial lesions that were not shown by CTA were later investigated and confirmed by DSA because of persistent hemorrhage. This might have been due to the higher contrast injection pressure used in DSA (direct arterial injection) being able to delineate smaller arterial lumens than CTA (venous injection). It may be hypothesized that CTA is a single morphologic snapshot in time and does not necessarily predict ongoing bleeding, and that disease progression between the two imaging sessions might have been the cause of the discrepancy between results, since DSA was performed 2-10 days after CTA in the present study. Therefore, a negative examination should not preclude further investigation if there is a high degree of clinical suspicion. Also, one arterial renal hemorrhage suspected on CTA was not shown by DSA, and this patient stabilized in the clinical follow-up without angiographic or surgical intervention. Spontaneous cessation of hemorrhage may have occurred prior to the DSA and should be a consideration when interpreting the DSA findings.

Another reason why CTA could not be a replacement for DSA is that the majority of renal artery lesions can be successfully controlled with endovascular treatment, which necessitates catheter angiography. The present study showed that all patients who underwent DSA directly without preceding CTA because of massive hematuria were confirmed to have positive findings of bleeding, indicating that in patients who are highly expected to be candidates for endovascular therapy, CTA would be an unnecessary extra step.

Despite these disadvantages, the detection rate of CTA for arterial renal hemorrhage was very high in the present study. CTA is a low-risk diagnostic technique without the need for arterial puncture or catheter manipulation, and it can also aid the interventional radiologist in immediately targeting the bleeding vessel during DSA. Therefore, it can be regarded as an excellent screening technique for arterial renal hemorrhage.

The present study has several limitations. First, not all patients who underwent multi-section CTA also underwent DSA. If the CTA results were negative, and the hematuria subsided following conservative measures, it was not necessary to proceed to DSA. However, these patients, who may be likely to provide negative results when examined by DSA, were not included in the study, resulting in underestimation of the specificity. Second, each CTA and DSA was evaluated by only one interventional radiologist and one urologist, and hence interobserver variability could not be calculated. This may be important in the reproducibility of the results of our study. Third, the study group had a broader range of different diagnoses, including iatrogenic injuries, vascular malformations and angiomyolipoma, and was more heterogeneous than that of Sadick et al (10).

In conclusion, CTA is a sensitive and accurate technique for the diagnosis of renal arterial bleeding, and is recommended 
as a road map for the management of these patients. However, it is not completely reliable in predicting the requirement for further treatment. In cases of massive renal hemorrhage, DSA with subsequent super selective embolization should be performed directly to avoid delayed treatment.

\section{Acknowledgements}

This study was supported by a grant from the National Natural Science Foundation of China (Grant No. 81101717), Specialized Research Fund for the Doctoral Program of Higher Education (Grant No. 20110101120111) and Zhejiang Provincial Medical Science Foundation of China (Grant No. 2013K YB086).

\section{References}

1. Brandes SB and McAninch JW: Urban free falls and patterns of renal injury: A 20-year experience with 396 cases. J Trauma 47 643-649, 1999.

2. Breyer BN, McAninch JW, Elliott SP and Master VA: Minimally invasive endovascular techniques to treat acute renal hemorrhage. J Urol 179: 2248-2252, 2008

3. Wang C, Mao Q, Tan F and Shen B: Superselective renal artery embolization in the treatment of renal hemorrhage. Ir J Med Sci 183: 59-63, 2013.
4. Young N, Chi KK, Ajaka J, McKay L, O'Neill D and Wong KP: Complications with outpatient angiography and interventional procedures. Cardiovasc Intervent Radiol 25: 123-126, 2002.

5. Waugh JR and Sacharias N: Arteriographic complications in the DSA era. Radiology 182: 243-246, 1992.

6. Kitase M, Mizutani M, Tomita H, Kono T, Sugie C and Shibamoto Y: Blunt renal trauma: Comparison of contrast-enhanced CT and angiographic findings and the usefulness of transcatheter arterial embolization. Vasa 36: 108-113, 2007.

7. Teksam M, McKinney A, Casey S, Asis M, Kieffer S and Truwit CL: Multi-section CT angiography for detection of cerebral aneurysms. AJNR Am J Neuroradiol 25: 1485-1492, 2004.

8. Chen CJ, Tseng YC, Lee TH, Hsu HL and See LC: Multisection CT angiography compared with catheter angiography in diagnosing vertebral artery dissection. AJNR Am J Neuroradiol 25: 769-774, 2004.

9. Edwards AJ, Wells IP and Roobottom CA: Multidetector row CT angiography of the lower limb arteries: A prospective comparison of volume-rendered techniques and intra-arterial digital subtraction angiography. Clin Radiol 60: 85-95, 2005.

10. Sadick M, Rohrl B, Schnulle P, Duber C and Diehl SJ: Multislice CT-angiography in percutaneous postinterventional hematuria and kidney bleeding: Influence of diagnostic outcome on therapeutic patient management. Preliminary results. Arch Med Res 38: 126-132, 2007.

11. Sommer CM, Stampfl U, Bellemann N, et al: Patients with life-threatening arterial renal hemorrhage: $\mathrm{CT}$ angiography and catheter angiography with subsequent superselective embolization. Cardiovasc Intervent Radiol 33: 498-508, 2010. 